\title{
Quantum criticality of semi-Dirac fermions in $2+1$ dimensions
}

\author{
Mikolaj D. Uryszek $\odot,{ }^{1}$ Elliot Christou, ${ }^{1}$ Akbar Jaefari $\odot,{ }^{2}$ Frank Krüger $\odot,{ }^{1,3}$ and Bruno Uchoa ${ }^{4}$ \\ ${ }^{1}$ London Centre for Nanotechnology, University College London, Gordon Street, London WC1H OAH, United Kingdom \\ ${ }^{2}$ Department of Physical and Biological Sciences, Western New England University, Springfield, Massachusetts 01119, USA \\ ${ }^{3}$ ISIS Facility, Rutherford Appleton Laboratory, Chilton, Didcot, Oxfordshire OX11 OQX, United Kingdom \\ ${ }^{4}$ Department of Physics and Astronomy, University of Oklahoma, Norman, Oklahoma 73069, USA
}

(Received 26 July 2019; published 1 October 2019)

\begin{abstract}
Two-dimensional semi-Dirac fermions are quasiparticles that disperse linearly in one direction and quadratically in the other. We investigate instabilities of semi-Dirac fermions toward charge and spin density wave and superconducting orders, driven by short-range interactions. We analyze the critical behavior of the Yukawa theories for the different order parameters using Wilson momentum shell renormalization group. We generalize to a large number $N_{f}$ of fermion flavors to achieve analytic control in $2+1$ dimensions and calculate critical exponents at one-loop order, systematically including $1 / N_{f}$ corrections. The latter depend on the specific form of the bosonic infrared propagator in $2+1$ dimensions, which needs to be included to regularize divergencies. The $1 / N_{f}$ corrections are surprisingly small, suggesting that the expansion is well controlled in the physical dimension. The order parameter correlations inherit the electronic anisotropy of the semi-Dirac fermions, leading to correlation lengths that diverge along the spatial directions with distinct exponents, even at the mean-field level. We conjecture that the proximity to the critical point may stabilize novel modulated order phases.
\end{abstract}

DOI: 10.1103/PhysRevB.100.155101

\section{INTRODUCTION}

Dirac fermions generically describe quasiparticles with relativistic dispersion in the vicinity of special points in the Brillouin zone [1-4]. In two spatial dimensions, the merging of two Dirac points results in a topological phase transition separating the semimetallic phase from a gapped insulating one $[5,6]$. At the boundary between the two phases, the system exhibits gapless "semi-Dirac" quasiparticle excitations [7] that disperse relativistically in one direction and quadratically in the other (see Fig. 1).

Based on density-functional calculations, semi-Dirac quasiparticles were predicted to occur in single layers of black phosphorus under strain [8]. Shortly after, they were observed when sprinkling potassium atoms onto single layers of black phosphorus [9], and more recently using surface doping [10]. Semi-Dirac electrons have also been predicted to occur in BEDT-TTF $\mathrm{I}_{3}$ salt under pressure [11], $\mathrm{VO}_{2} / \mathrm{TO}_{2}$ heterostructures $[12,13]$, and strained honeycomb lattices [12]. Recently, it has been suggested [14,15] that second-neighbor repulsions between Dirac fermions on the honeycomb lattice can lead to a metallic charge density wave (CDW) state that breaks lattice symmetries and exhibits semiDirac quasiparticle excitations. Semi-Dirac fermions have strongly anisotropic hydrodynamic transport properties [16]; e.g., the electrical conductivity is metallic in one direction and insulating in the other direction. Even more strikingly, one of the electronic sheer viscosity components vanishes at zero temperature, leading to a generalization of the previously conjectured lower bound for the viscosity to entropy density ratio [16].

Nodal semimetals with pointlike Fermi surfaces represent the simplest example of fermionic quantum criticality, driven by strong short-range repulsive interactions [17]. The symmetry breaking leads to the opening of a gap in the fermion spectrum and therefore goes hand in hand with a semimetalto-insulator transition. In the purely relativistic case of Dirac fermions it is well understood [18-21] that the universal behavior is described by the Gross-Neveu-Yukawa theory $[22,23]$ of chiral symmetry breaking. The coupling between the order parameter fields and the gapless Dirac fermions leads to novel fermion-induced critical behavior that falls outside the Landau-Ginzburg-Wilson paradigm of a pure order parameter description. Ultimately, the study of quantum phase transitions in nodal semimetals might serve as a stepping stone toward an understanding of quantum criticality in metals with extended Fermi surfaces.

The nontrivial scaling of the quasiparticle kinetic energy of semi-Dirac fermions gives rise to novel universal behavior [24-27]. Moreover, it is expected that the inherent electronic anisotropy will be reflected in strongly anisotropic order parameter correlations. This can have profound effects on the nature of broken-symmetry states. For instance, in gapped superconducting phases, it has been suggested [28] that an applied magnetic field may lead to the formation of a novel smectic phase with a stripe pattern of flux domains near the quantum critical point.

Accessing the universal critical behavior of twodimensional semi-Dirac fermions has proven difficult. Because of the different dispersion along the $k_{x}$ and $k_{y}$ directions, the generalization to arbitrary dimension and consecutive $\epsilon$ expansion below an upper critical dimension is subtle and not uniquely defined. For a model with $d_{\mathrm{L}}$ linear and $d_{\mathrm{Q}}>0$ quadratic momentum directions, the interactions become marginal at $2 d_{\mathrm{L}}+d_{\mathrm{Q}}=4$, suggesting that the universal critical behavior of two-dimensional semi-Dirac 


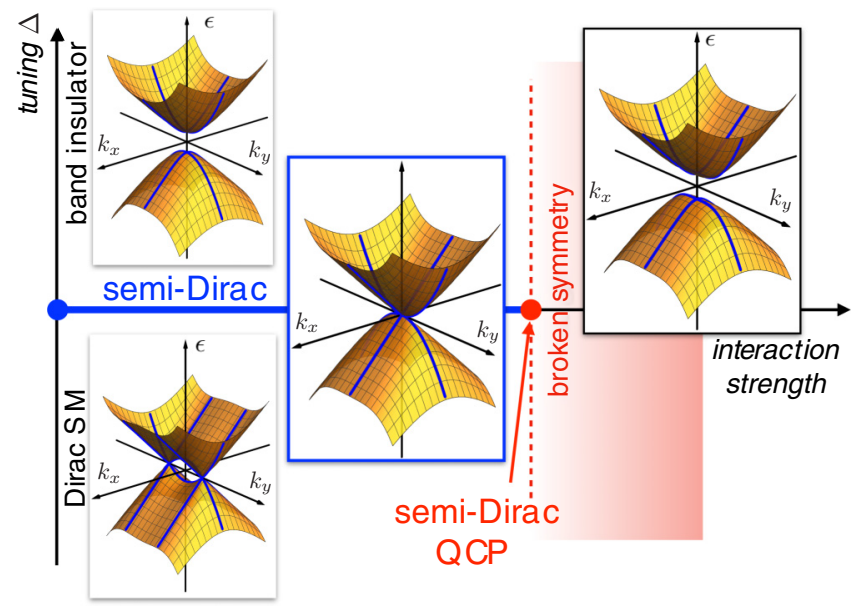

FIG. 1. Schematic phase diagram. As a function of the band tuning parameter $\Delta$ the system undergoes a topological Lifshitz transition between a Dirac semimetal (with a pair of Dirac cones) and a band insulator. At the transition point the system exhibits gapless "semi-Dirac" quasiparticle excitations. Sufficiently strong short-range interactions lead to antiferromagnetic, CDW, or superconducting order, depending on the type of interaction. The symmetry breaking is associated with the opening of a gap in the semi-Dirac spectrum.

fermions $\left(d_{\mathrm{L}}=d_{\mathrm{Q}}=1\right)$ could be accessible within an $\epsilon$ expansion with $d_{\mathrm{L}}=1$ and $d_{\mathrm{Q}}=2-\epsilon$ [27]. This expansion results in a nonanalytic $\sim \epsilon \ln \epsilon$ dependence of the anomalous dimensions of the fermion and order parameter fields [27]. The nonmonotonic behavior and vanishing of the corrections at $\epsilon=1$, nevertheless, could indicate that the semi-Dirac case lies outside the validity of the expansion.

In a complementary approach [26] generic short-range four-fermion interactions were analyzed in two spatial dimensions with a generalized dispersion $k_{y}^{2 n}$ in the nonrelativistic direction. This construction allows for a controlled ascent from one dimension $(n \rightarrow \infty)$. At finite $n$, interactions are rendered irrelevant at weak coupling, but key aspects of one-dimensional physics such as spin-charge separation are preserved. Quantum fluctuations beyond 1D, controlled by $\sim 1 / n$, enter the renormalization group (RG) through loop integrations that involve the dispersion along $k_{y}$.

In this paper we revisit the criticality of semi-Dirac fermions in $2+1$ dimensions. We avoid tuning the dimensionality or form of the dispersion but instead introduce a large number $N_{f}$ of fermion flavors for analytical control. The additional fermion flavors are not involved in the symmetry breaking and remain degenerate across the quantum phase transition. We use a one-loop, momentum-shell RG to calculate critical exponents to order $1 / N_{f}$. A similar procedure was used to analyze the effects of weak, long-range Coulomb interactions between semi-Dirac fermions in two and three spatial dimensions [25,29]. Here we focus on strong shortrange interactions that drive antiferromagnetic, $\mathrm{CDW}$, and superconducting instabilities. This study is motivated by the leading order RG analysis of Ref. [26], which deemed these to be the dominant instabilities in the extended Hubbard model with on-site (attractive and repulsive) and nearest-neighbor repulsive interactions. These broken-symmetry phases possess a fully gapped quasiparticle spectrum and hence maximize the condensation energy gain. The results can be readily compared to the analogous large- $N_{f}$ analysis of relativistic $2 \mathrm{D}$ Dirac fermions [18,21], unraveling the effects of the peculiar form of the dispersion on the universal behavior.

We find that the $1 / N_{f}$ corrections to critical exponents are very small and considerably smaller than for the case of $2 \mathrm{D}$ Dirac fermions, suggesting that the expansion is well controlled. These $1 / N_{f}$ corrections depend on the specific form of the bosonic infrared (IR) propagator in $2+1$ dimensions, which needs to be included to regularize divergencies. As expected, we find that the order parameter correlations inherit the intrinsic anisotropy of the system; e.g., the correlation lengths along different spatial directions diverge with different powers. We conjecture that this behavior could help stabilize exotic modulated ordered phases near the quantum critical point.

Even the $N_{f} \rightarrow \infty$ results are significantly different from the 2D Dirac case. This can be understood by analyzing the mean-field Ginzburg-Landau free energy that is obtained from integrating out the fermions in the broken-symmetry state. Spatial anisotropies are encoded in nonanalytic gradient terms. We find that the critical exponents derived from the RG for $N_{f} \rightarrow \infty$ are in agreement with ones obtained from the mean-field Ginzburg-Landau functional, suggesting that hyperscaling relations are satisfied.

The outline of the paper is a follows: In Sec. II we derive the effective Yukawa actions for spin, charge, and superconducting instabilities. In Sec. III we explain the Wilson RG procedure, derive the one-loop RG equations in the large- $N_{f}$ limit, and compute critical exponents to order $1 / N_{f}$. The nonanalytic structure of the mean-field theory is discussed in Sec. IV. Finally, in Sec. V we summarize our results, compare them to those obtained from different expansion schemes $[26,27]$, and discuss their implications.

\section{EFFECTIVE FIELD THEORY}

In this section we motivate the effective low-energy field theory for different instabilities of semi-Dirac fermions in $2+1$ dimensions. The part of the action describing noninteracting fermions is given by

$$
\begin{aligned}
\mathcal{S}_{\psi}= & \sum_{n=1}^{N_{f}} \int_{\vec{k}} \overline{\boldsymbol{\psi}}_{n}(\vec{k}) \boldsymbol{s}^{0} \otimes\left[-i k_{0} \boldsymbol{\sigma}^{0}+v k_{x} \boldsymbol{\sigma}^{x}\right. \\
& \left.+\left(\frac{k_{y}^{2}}{2 m}+\Delta\right) \boldsymbol{\sigma}^{y}\right] \boldsymbol{\psi}_{n}(\vec{k}),
\end{aligned}
$$

where $\vec{k}=\left(k_{0}, \boldsymbol{k}\right)=\left(k_{0}, k_{x}, k_{y}\right)$, with $k_{0}$ the Matsubara frequency, and $\int_{\vec{k}} \equiv \int \frac{d^{3} \vec{k}}{(2 \pi)^{3}}$, subject to an ultraviolet cutoff $\Lambda$. The two sets of Pauli matrices $\boldsymbol{s}^{i}$ and $\boldsymbol{\sigma}^{i}$ (i=0 for identities) act on the spin and sublattice spaces, respectively, and the fermionic Grassmann fields $\psi_{n}(\vec{k})$ are 4-component spinors. We have generalized the action by introducing $N_{f}$ flavors or copies of Grassmann fields, labeled by $n$. 
From the poles of the corresponding Green's function we obtain the electron dispersion,

$$
\epsilon(\boldsymbol{k})= \pm \sqrt{\left(v k_{x}\right)^{2}+\left(\frac{k_{y}^{2}}{2 m}+\Delta\right)^{2}},
$$

which is degenerate in spin $s=\uparrow, \downarrow$ and fermion flavor $n$. The effect of the tuning parameter $\Delta$ on the electron dispersion is illustrated in Fig. 1. For $\Delta<0$ the dispersion contains two relativistic Dirac points $\boldsymbol{K}_{ \pm}=(0, \pm \sqrt{2 m(-\Delta)})$, while for $\Delta>0$ the dispersion has an energy gap $\Delta$. Hence $\Delta$ tunes a transition between a Dirac semimetal and a band insulator. At $\Delta=0$, the system undergoes a topological Lifshitz transition, corresponding to the merging of two Dirac points. At this point the system exhibits semi-Dirac quasiparticle excitations.

We aim to describe interaction-driven quantum phase transition of semi-Dirac fermions. Enforcing $\Delta=0$ while increasing the strength of interactions requires fine tuning. Depending on the experimental system, this may be achieved with strain, pressure, or surface doping [8-13].

\section{A. CDW and SDW instabilities}

To study the criticality of semi-Dirac fermions subject to local interactions we use the Yukawa formalism. This amounts to performing a Hubbard-Stratonovich transformation of a generic four-fermion interaction vertex in the appropriate order parameter channel. Here we do not address the question of phase competition but instead focus on a particular type of symmetry breaking, and assume an underlying fermion interaction that would stabilize this order.

In the case of CDW and spin density wave (SDW) instabilities, the Yukawa coupling between the order parameter fields $\phi_{i}(\vec{k})$ and the fermions $\psi_{n}(\vec{k})$ is given by

$$
\mathcal{S}_{g}=g \sum_{n=1}^{N_{f}} \sum_{i}^{N_{b}} \int_{\vec{k}, \vec{q}} \phi_{i}(\vec{q}) \overline{\boldsymbol{\psi}}_{n}(\vec{k}) \mathbf{Y}_{i} \boldsymbol{\psi}_{n}(\vec{k}+\vec{q}),
$$

where

$$
\mathbf{Y}_{i}= \begin{cases}s^{0} \otimes \boldsymbol{\sigma}^{z}, & \text { CDW: } i=0, \\ \boldsymbol{s}^{i} \otimes \boldsymbol{\sigma}^{z}, & \text { SDW: } i=\{x, y, z\} .\end{cases}
$$

Both Yukawa couplings anticommute with the noninteracting action. That gives rise to a fully gapped quasiparticle dispersion in the broken-symmetry phase,

$$
\epsilon(\boldsymbol{k})= \pm \sqrt{\left(v k_{x}\right)^{2}+\left(k_{y}^{2} / 2 m\right)^{2}+|g \boldsymbol{\phi}|^{2}},
$$

maximizing the condensation energy gain.

In spite of their name, the CDW and the SDW states describe a staggered field in the pseudospin space $\left(\sigma^{z}\right)$, which could in principle be any generic quantum number in the original lattice model, such as sublattice, valley, or orbital quantum numbers. In the CDW state, the order parameter is a scalar $\left(N_{b}=1\right)$, whereas in the SDW, which in this language is equivalent to antiferromagnetism, $N_{b}=3$.

In addition to a $\phi^{2}$ term that arises from the HubbardStratonovich transformation, the successive elimination of high-energy fermion modes under the renormalization group will generate gradient terms, as well as a $\phi^{4}$ vertex. The resulting Ginzburg-Landau functional for the order parameter is given by $\mathcal{S}_{\phi}+\mathcal{S}_{\lambda}$ with

$$
\begin{gathered}
\mathcal{S}_{\boldsymbol{\phi}}=\frac{1}{2} \int_{\vec{q}}\left(c_{0}^{2} q_{0}^{2}+c_{x}^{2} q_{x}^{2}+c_{y}^{2} q_{y}^{2}+m_{\phi}^{2}\right)|\boldsymbol{\phi}(\vec{q})|^{2}, \\
\mathcal{S}_{\lambda}=\lambda \int_{\boldsymbol{r}, \tau}|\boldsymbol{\phi}(\boldsymbol{r}, \tau)|^{4},
\end{gathered}
$$

where the integral of the $\phi^{4}$ vertex runs over real space $\boldsymbol{r}=$ $(x, y)$ and imaginary time $\tau$. The order parameter mass term $m_{\phi}$ is the tuning parameter for the broken-symmetry state: At the critical point $m_{\phi}^{2}=0$. To summarize, the effective field theory describing the criticality of semi-Dirac fermions in 2 +1 dimensions is given by the sum of four terms,

$$
\mathcal{S}=\mathcal{S}_{\psi}+\mathcal{S}_{\phi}+\mathcal{S}_{g}+\mathcal{S}_{\lambda}
$$

There is a caveat, however. As we will discuss in Sec. III, the bosonic propagator $G_{\phi}(\vec{q})$ develops an unphysical singularity under the renormalization group scheme. It is therefore necessary to regularize this divergence by including an IR contribution in $\mathcal{S}_{\phi}$.

\section{B. Superconducting instability}

We also analyze a possible instability in the superconducting channel. In that case, we initially consider a generic Hamiltonian $\mathcal{H}_{0}(\boldsymbol{p})$ with semi-Dirac quasiparticles that preserves time-reversal symmetry (TRS), such that $\mathcal{T} \mathcal{H}_{0}(\boldsymbol{p}) \mathcal{T}^{-1}=\mathcal{H}_{0}(\boldsymbol{p})$, with $\boldsymbol{p}$ the momentum away from the center of the zone. We restrict our attention to families of TRS Hamiltonians that support an isotropic pairing gap around the semi-Dirac points. That is allowed when pairing occurs across two semi-Dirac points sitting in opposite sides of the Brillouin zone, such that each Cooper pair has zero total momentum. A concrete example of a TRS tight-binding Hamiltonian with a pair of semi-Dirac points can be found in Ref. [28].

Due to TRS, the generic Hamiltonian can be written in a Bogoliubov-de Gennes basis $\left(c_{p, s}, c_{-p,-s}^{\dagger}\right)$ with $s$ the spin index as

$$
\mathcal{H}_{\mathrm{BdG}}(\boldsymbol{p})=\mathcal{H}_{0}(\boldsymbol{p}) \otimes \tau^{z},
$$

where $\tau^{z}$ is a Pauli matrix in the Nambu space. Expanding this Hamiltonian around one of the semi-Dirac points described, the action in the Nambu basis is

$$
\begin{aligned}
\mathcal{S}_{\psi}= & \sum_{n=1}^{N_{f}} \int_{\vec{k}} \overline{\boldsymbol{\psi}}_{n}(\vec{k}) \boldsymbol{s}^{0} \otimes\left[-i k_{0} \boldsymbol{\sigma}^{0} \otimes \boldsymbol{\tau}^{0}+v k_{x} \boldsymbol{\sigma}^{x} \otimes \boldsymbol{\tau}^{z}\right. \\
& \left.+\left(\frac{k_{y}^{2}}{2 m}+\Delta\right) \boldsymbol{\sigma}^{y} \otimes \boldsymbol{\tau}^{z}\right] \boldsymbol{\psi}_{n}(\vec{k}),
\end{aligned}
$$

where we enforce $\Delta=0$ at the fixed point. The expansion around the opposite Dirac point gives an equivalent copy of the action above, which can be accounted for in the fermionic degeneracy $N_{f}$.

The Yukawa coupling between the complex, twocomponent $\left(N_{b}=2\right)$ order parameter of the $s$-wave superconductor and the fermions is given by Eq. (3) with the coupling matrix

$$
\mathbf{Y}_{i}=\boldsymbol{s}^{0} \otimes \boldsymbol{\sigma}^{0} \otimes \boldsymbol{\tau}^{i}, \quad \text { SC: } i=\{x, y\},
$$


in the enlarged Nambu space. This term anticommutes with the noninteracting action, leading to the opening of a gap in the semi-Dirac spectrum. $\mathcal{S}_{\boldsymbol{\phi}}$ and $\mathcal{S}_{\lambda}$ take the same form as in the CDW/SDW case.

\section{RENORMALIZATION GROUP ANALYSIS}

In this section, we outline the RG approach used to analyze the universal critical behavior of the Yukawa actions derived in Sec. II for CDW, SDW, and superconducting instabilities. In the case of the superconducting instability, one is required to include the Nambu space, as indicated in the action (10), and sum over half the number of states in the trace to avoid double counting. For simplicity, we omit any explicit mention to the Nambu space, which can be trivially incorporated for the SC instability.

The universal critical behavior, e.g., the critical exponents, should not depend on the cutoff scheme. In the following we treat frequency and momentum on an equal footing and impose the ultraviolet (UV) cutoff $\Lambda$ in $2+1$ dimensional momentum-frequency space,

$$
\int_{\vec{k}} \equiv \int \frac{d^{3} \vec{k}}{(2 \pi)^{3}} \theta\left[\Lambda-\sqrt{k_{0}^{2}+\epsilon^{2}(\boldsymbol{k})}\right],
$$

where the electron dispersion $\epsilon(\boldsymbol{k})$ is defined in Eq. (2) with $\Delta=0$. We will analyze the RG flow of the coupling constants when successively integrating out high-energy modes with momenta and frequencies from the infinitesimal shell,

$$
\Lambda e^{-z d \ell}<\sqrt{k_{0}^{2}+\epsilon^{2}(\boldsymbol{k})}<\Lambda
$$

followed by a rescaling of frequency and momenta at each step,

$$
k_{0}=k_{0}^{\prime} e^{-z d \ell}, k_{x}=k_{x}^{\prime} e^{-z d \ell}, k_{y}=k_{y}^{\prime} e^{-z_{y} d \ell} .
$$

In the definitions of the $2+1$ dimensional shell (13) and the rescaling (14), frequency $k_{0}$ and the momentum $k_{x}$ along the relativistic direction are treated on an equal footing and are both rescaled with a dynamical exponent $z$ relative to the $k_{y}$ direction. One might view this as having one spacelike and two timelike directions. For greater clarity, we have introduced a scaling dimension $z_{y}$ of the momentum $k_{y}$, which we will set to 1 later. Under successive mode decimation and rescaling, the cutoff remains invariant if

$$
z=2 z_{y}
$$

The shell integration is performed using the coordinate transformation

$$
\begin{aligned}
k_{0} & =\epsilon \sin \theta \cos \phi, \\
v k_{x} & =\epsilon \sin \theta \sin \phi, \\
\frac{k_{y}^{2}}{2 m} & =\epsilon \cos \theta,
\end{aligned}
$$

with $\epsilon \in\left[\Lambda e^{-z d \ell}, \Lambda\right], \theta \in\left[0, \frac{\pi}{2}\right]$, and $\phi \in[0,2 \pi]$. The Jacobian of the transformation is

$$
\rho(\epsilon, \theta, \phi)=\frac{\sqrt{2 m}}{2 v} \frac{\sin \theta}{\sqrt{\cos \theta}} \epsilon^{3 / 2} .
$$

\section{A. Tree level scaling}

Let us first consider the consequences of the rescaling of frequency and momenta (14) on the free-fermion action $\mathcal{S}_{\psi}$ (1). Since frequency $k_{0}$ and momentum $k_{x}$ have the same scaling dimensions, $\left[k_{0}\right]=\left[k_{x}\right]=z$, the velocity $v$ of the relativistic dispersion along $k_{x}$ does not flow. The relation $z=2 z_{y}$ ensures that the mass $m$ associated with the quadratic $k_{y}$ dispersion remains constant at the tree level.

In order to keep the overall prefactor of $\mathcal{S}_{\psi}$ constant, we rescale the fermion fields as

$$
\boldsymbol{\psi}_{n}(\vec{k})=\boldsymbol{\psi}_{n}^{\prime}\left(\vec{k}^{\prime}\right) e^{d \ell\left(3 z+z_{y}-\eta_{\psi}\right) / 2} .
$$

Here $\eta_{\psi}$ is the anomalous dimension of the fermion fields, which will be used to absorb the renormalization due to the decimation of high-energy modes.

Let us turn our attention to the quadratic bosonic action $\mathcal{S}_{\phi}$ (6). The distinct scaling dimensions of momenta imply that the coefficients $c_{0}^{2}$ and $c_{x}^{2}$ will scale differently from $c_{y}^{2}$. If we rescale the bosonic fields as

$$
\boldsymbol{\phi}(\vec{q})=\boldsymbol{\phi}^{\prime}\left(\vec{q}^{\prime}\right) e^{d \ell\left(2 z+3 z_{y}-\eta_{\phi}\right) / 2},
$$

where $\eta_{\phi}$ is the anomalous dimension of the $\phi$ field, the $c_{y}^{2}$ coefficient does not flow at tree level. Under this rescaling both $c_{0}^{2}$ and $c_{x}^{2}$ are irrelevant at tree level with scaling dimensions $\left[c_{0}^{2}\right]=\left[c_{x}^{2}\right]=-z$. Both parameters flow to zero and can be omitted from the bare bosonic propagator. This omission poses problems, as the propagator no longer depends on $q_{0}$ and $q_{x}$, leading to unphysical divergencies in the infrared (IR) limit. That issue however can be fixed with an IR regularization, as discussed next in Sec. III B.

The above field rescaling leads to the following tree level scaling dimensions for the Yukawa and the $\phi^{4}$ couplings,

$$
[g]=\frac{z_{y}}{2}, \quad[\lambda]=-z_{y} .
$$

This portrays that the $\boldsymbol{\phi}^{4}$ term is irrelevant and can be discarded, while the Yukawa coupling is a relevant perturbation at tree level.

\section{B. Infrared regularization}

In order to regularize unphysical divergencies of the bosonic propagator in the limit $q_{y} \rightarrow 0$, we augment it by an IR contribution [30-32]. As pointed out before [25], in $2+1$ dimensions the bosonic propagator has different asymptotic forms in the UV and IR limits.

Since the RG flow is generated by a successive integration of modes from a shell near the UV cutoff, the IR contribution is not generated or renormalized under the RG. Instead it needs to be computed separately by integrating the fermion polarization (see Fig. 2) over the entire frequency and momentum range up to the infinitesimal shell [25],

$$
\Pi_{i j}(\vec{q})=\frac{g^{2}}{2} \operatorname{Tr} \int_{\vec{k}}^{<} \mathbf{Y}_{i} \mathbf{G}_{\psi}(\vec{k}) \mathbf{Y}_{j} \mathbf{G}_{\psi}(\vec{k}+\vec{q}),
$$

where $\mathbf{Y}_{i}$ is the Yukawa coupling for a given instability, as defined in Eqs. (4) and (11), and

$$
\mathbf{G}_{\psi}(\vec{k})=\boldsymbol{s}^{0} \otimes \frac{i k_{0} \boldsymbol{\sigma}^{0}+v k_{x} \boldsymbol{\sigma}^{x}+\frac{k_{y}^{2}}{2 m} \boldsymbol{\sigma}^{y}}{k_{0}^{2}+\left(v k_{x}\right)^{2}+k_{y}^{4} /(2 m)^{2}}
$$

is the fermionic propagator. 


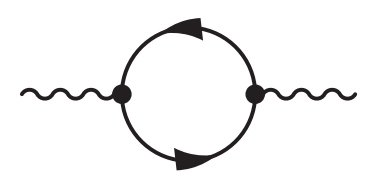

FIG. 2. Polarization bubble diagram describing the IR regulator of the bosonic propagator and the self-energy correction of the bosons in the momentum shell.

For the multicomponent order parameters of the SDW and superconducting phases, the polarization is diagonal, $\Pi_{i j}(\vec{q})=\Pi(\vec{q}) \delta_{i j}$, reflecting the underlying $\mathrm{O}(3)$ and $\mathrm{U}(1)$ symmetries. In the limit of small $q_{0}, q_{x}$ and at $q_{y}=0$, the leading term is [25]

$$
\Pi_{\mathrm{IR}}(\vec{q})=N_{f} g^{2} \frac{\sqrt{2 m}}{v}\left(q_{0}^{2}+v^{2} q_{x}^{2}\right)^{\frac{1}{4}},
$$

where $N_{f}$ is the number of fermion flavors. This contribution to the kernel of $\mathcal{S}_{\phi}$ regularizes the bosonic propagator in the IR at the critical surface $\left(m_{\phi}^{2}=0\right)$,

$$
G_{\phi}^{-1}(\vec{q})=N_{f} g^{2} \frac{\sqrt{2 m}}{v}\left(q_{0}^{2}+v^{2} q_{x}^{2}\right)^{\frac{1}{4}}+c_{y}^{2} q_{y}^{2} .
$$

\section{Self-energy and vertex corrections}

Using the propagators (22) and (24) for fermionic and bosonic fields, respectively, we can now go beyond the tree level scaling and extract one-loop corrections to the propagators and the Yukawa coupling. As mentioned before, the bosonic $\phi^{4}$ is irrelevant and can be dropped.

We first concern ourselves with the one-loop renormalization of the regularized bosonic propagator (24). The only component that is of interest is in the $q_{y}$ direction as the dependence on linear momentum and frequency directions in the propagator comes from the IR, which is not renormalized under the RG. The one-loop bosonic self-energy is depicted in Fig. 2 and takes the form

$$
\Pi^{>}(\vec{q})=\frac{g^{2}}{2} \operatorname{Tr} \int_{\vec{k}}^{>} \mathbf{Y}_{i} \mathbf{G}_{\psi}(\vec{k}) \mathbf{Y}_{i} \mathbf{G}_{\psi}(\vec{k}+\vec{q}),
$$

where $\int_{\vec{k}}$ means integration over the UV modes within the frequency-momentum shell of width $\Lambda z d \ell$, as defined in Eq. (13). Note that in the above expression, the order parameter field component $i$ is not summed over and that the result is the same for all components. The leading terms of the self-energy have the form $\Pi^{>}(\vec{q})=\Pi_{0} q_{0}^{2}+\Pi_{x} q_{x}^{2}+$ $\Pi_{y} q_{y}^{2}+\Pi_{m_{\phi}^{2}}$. Expanding the fermionic propagator to second order in $q_{y}$, then performing the coordinate transformation (16) and integrating over the energy shell, we find that the renormalization of the $c_{y}^{2}$ coefficient is given by

$$
d\left(c_{y}^{2}\right)=2 N_{f} \frac{11}{21 \pi^{2}} \frac{\sqrt{2 m}}{v} \frac{g^{2}}{\sqrt{\Lambda}} z d \ell \equiv \Pi_{y} z d \ell .
$$

Although the mass of the bosons $m_{\phi}^{2}$ also runs in the RG flow, for now it will be fine tuned to zero at the critical surface. We will address the renormalization of $m_{\phi}^{2}$ in detail later on in Secs. IIIF and III G, when we examine the vicinity of the quantum multicritical point. (a)

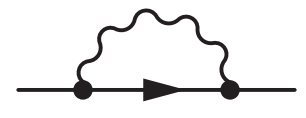

(b)

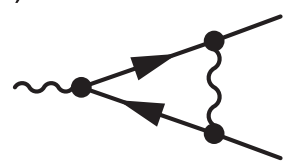

FIG. 3. (a) Self-energy correction to the fermionic propagator in one loop. (b) Vertex correction diagram to the Yukawa coupling. The bosonic propagator is represented by the wavy line while the fermionic propagator by the straight line.

Next, we turn our attention to the one-loop fermionic selfenergy [Fig. 3(a)], which is equal to

$$
\boldsymbol{\Sigma}(\vec{k})=-g^{2} \sum_{i}^{N_{b}} \int_{\vec{q}}^{>} G_{\phi}(\vec{q}) \mathbf{Y}_{i} \mathbf{G}_{\psi}(\vec{k}+\vec{q}) \mathbf{Y}_{i} .
$$

After shell integration, it takes the form

$$
\boldsymbol{\Sigma}(\vec{k})=\boldsymbol{s}^{0} \otimes\left[\Sigma_{0}\left(k_{0} \boldsymbol{\sigma}^{0}+v k_{x} \boldsymbol{\sigma}^{x}\right)+\Sigma_{y} \frac{k_{y}^{2}}{2 m} \boldsymbol{\sigma}^{y}\right] z d \ell,
$$

where

$$
\begin{aligned}
& \Sigma_{0}=\frac{N_{b}}{2 N_{f}} F_{1}\left(\frac{2 N_{f} \sqrt{2 m} g^{2}}{v c_{y}^{2} \sqrt{\Lambda}}\right), \\
& \Sigma_{y}=\frac{N_{b}}{2 N_{f}} F_{2}\left(\frac{2 N_{f} \sqrt{2 m} g^{2}}{v c_{y}^{2} \sqrt{\Lambda}}\right),
\end{aligned}
$$

with $\Sigma_{x}=\Sigma_{0}$, and

$$
\begin{gathered}
F_{1}(x)=\frac{1}{4 \pi^{2}} \int_{0}^{\frac{\pi}{2}} d \theta \frac{(\cos \theta)^{\frac{3}{2}} \sin \theta}{x^{-1} \cos \theta+\sqrt{\sin \theta}}, \\
F_{2}(x)=\frac{1}{4 \pi^{2}} \int_{0}^{\frac{\pi}{2}} d \theta \frac{\cos 2 \theta+2 \cos 4 \theta}{x^{-1} \cos \theta+\sqrt{\sin \theta}} \frac{\sin \theta}{\sqrt{\cos \theta}}
\end{gathered}
$$

are defined as integral functions.

The renormalization of the Yukawa vertex at one-loop order is obtained from the diagram shown in Fig. 3(b) with the external frequencies and momenta set to zero,

$$
\boldsymbol{\Gamma}_{i}=g^{3} \sum_{j}^{N_{b}} \int_{\vec{q}}^{>} G_{\phi}(\vec{q}) \mathbf{Y}_{j} \mathbf{G}_{\psi}(\vec{q}) \mathbf{Y}_{i} \mathbf{G}_{\psi}(\vec{q}) \mathbf{Y}_{j} .
$$

The matrix $\boldsymbol{\Gamma}_{i}$ is proportional to the Yukawa matrix $\mathbf{Y}_{i}, \boldsymbol{\Gamma}_{i}=$ $g \Omega \mathbf{Y}_{i} z d \ell$, where we have absorbed a factor of $g^{2}$ in the definition of $\Omega$. Performing the shell integral we obtain

$$
\Omega=-\frac{2-N_{b}}{2 N_{f}} F_{3}\left(\frac{2 N_{f} \sqrt{2 m} g^{2}}{v c_{y}^{2} \sqrt{\Lambda}}\right)
$$

with

$$
F_{3}(x)=\frac{1}{4 \pi^{2}} \int_{0}^{\frac{\pi}{2}} d \theta \frac{1}{x^{-1} \cos \theta+\sqrt{\sin \theta}} \frac{\sin \theta}{\sqrt{\cos \theta}} .
$$

Note that the vertex correction to the Yukawa coupling $g$ has opposite sign for the CDW $\left(N_{b}=1\right)$ and SDW $\left(N_{b}=3\right)$ instabilities and vanishes in the case of a superconductor $\left(N_{b}=2\right)$, as reported in previous studies [27]. 


\section{RG equations}

As a first step we analyze the $\mathrm{RG}$ flow of the fermion propagator $\mathcal{S}_{\psi}(1)$, combining the contributions from the selfenergy loop integral $\Sigma(27)$ and the rescaling outlined in Sec. III A. Since $\Sigma_{0}=\Sigma_{x}$, the coefficients of the $k_{0}$ and $k_{x}$ terms are renormalized in the same way. We can keep the prefactor of the two terms constant if we define the anomalous dimension of the fermion fields as

$$
\eta_{\psi}=z \Sigma_{0}
$$

The resulting $\mathrm{RG}$ equation for $m^{-1}$ is then given by

$$
\frac{d\left(m^{-1}\right)}{d \ell}=m^{-1}\left(z-2 z_{y}+z \Sigma_{y}-z \Sigma_{0}\right)
$$

with the self-energy corrections $\Sigma_{0}$ and $\Sigma_{y}$ given in Eqs. (29) and (30), respectively. In order to keep the full fermion propagator invariant under the RG flow, we therefore require that

$$
2 z_{y}=z\left(1+\Sigma_{y}-\Sigma_{0}\right)
$$

In the bosonic propagator $\mathcal{S}_{\boldsymbol{\phi}}(24)$, the coefficient $c_{y}^{2}$ is renormalized,

$$
\frac{d\left(c_{y}^{2}\right)}{d \ell}=c_{y}^{2}\left(z \Pi_{y}-\eta_{\phi}\right)
$$

but can be kept fixed if we chose the anomalous dimension of the bosonic order parameter to be

$$
\eta_{\phi}=z \Pi_{y}
$$

where the polarization shell integral is defined in Eq. (26).

Finally, the RG equation for the Yukawa coupling $g$ at oneloop order is given by

$$
\frac{d g}{d \ell}=g\left(\frac{z_{y}}{2}-\eta_{\psi}-\frac{1}{2} \eta_{\phi}+z \Omega\right),
$$

with $\Omega$ defined in Eq. (34).

\section{E. Fixed point and $1 / N_{f}$ expansion}

With the above choices of $\eta_{\psi}, \eta_{\phi}$, and $z$ (we fix $z_{y}=1$ in what follows) the coefficients entering the propagators do not flow and can be set to 1, without loss of generality. Moreover it is convenient to define the rescaled Yukawa coupling

$$
\tilde{g}^{2}=\frac{2 N_{f} g^{2}}{\sqrt{\Lambda}}
$$

as this combination enters in the argument of the functions $F_{i}$. These are smooth functions of $\tilde{g}^{2}$, as shown in Fig. 4. In terms of the $F$ functions, the RG equation for the rescaled Yukawa coupling is given by

$$
\frac{d \tilde{g}^{2}}{d \ell}=\tilde{g}^{2}\left\{1-z\left[\frac{11}{21 \pi^{2}} \tilde{g}^{2}+\frac{N_{b}}{N_{f}} F_{1}\left(\tilde{g}^{2}\right)+\frac{2-N_{b}}{N_{f}} F_{3}\left(\tilde{g}^{2}\right)\right]\right\}
$$

and from Eq. (38)

$$
2=z\left\{1-\frac{N_{b}}{2 N_{f}}\left[F_{1}\left(\tilde{g}^{2}\right)-F_{2}\left(\tilde{g}^{2}\right)\right]\right\} .
$$

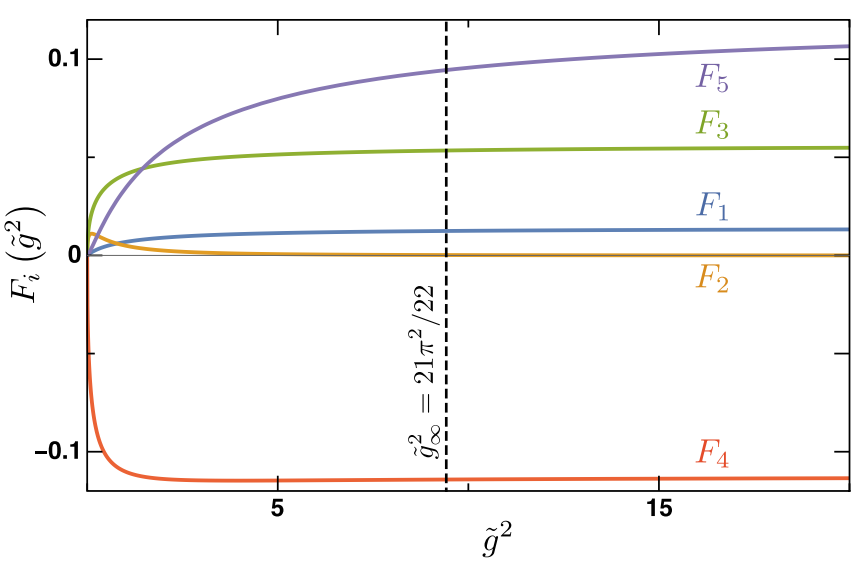

FIG. 4. The integrals $F_{i}\left(\tilde{g}^{2}\right)$ as a function of the dimensionless Yukawa coupling $\tilde{g}^{2}=2 N_{f} g^{2} / \sqrt{\Lambda}$. To order $1 / N_{f}$, the critical exponents at one-loop order depend on the values of $F_{i}$ evaluated at the critical point in the $N_{f} \rightarrow \infty$ limit, $\tilde{g}_{\infty}^{2}=\lim _{N_{f} \rightarrow \infty} \tilde{g}_{*}^{2}=21 \pi^{2} / 22$.

The fixed point is obtained from $d \tilde{g}^{2} / d \ell=0$. In the limit $N_{f} \rightarrow \infty, z=2$ and the fixed point is at

$$
\tilde{g}_{\infty}^{2}=\frac{21 \pi^{2}}{22} .
$$

In order to obtain the leading $1 / N_{f}$ correction to the fixed point we make an ansatz

$$
\tilde{g}_{*}^{2}=\tilde{g}_{\infty}^{2}+\frac{\delta}{N_{f}}+O\left(1 / N_{f}^{2}\right)
$$

Since all the $F$ functions have $1 / N_{f}$ prefactors, we can replace their arguments with $\tilde{g}_{\infty}^{2}$ and define

$$
\alpha_{i}:=F_{i}\left(\tilde{g}_{\infty}^{2}\right) \text {. }
$$

To order $1 / N_{f}$ we obtain

$$
z=2+\frac{N_{b}}{N_{f}}\left(\alpha_{1}-\alpha_{2}\right)
$$

for the scaling dimension $z$ at the fixed point, and

$$
\frac{\tilde{g}_{*}^{2}}{\tilde{g}_{\infty}^{2}}=1-\frac{N_{b}}{2 N_{f}}\left(5 \alpha_{1}-\alpha_{2}\right)-2 \frac{2-N_{b}}{N_{f}} \alpha_{3}
$$

for the Yukawa coupling at the fixed point. Finally, the anomalous dimensions at the critical point are

$$
\eta_{\psi}=\frac{N_{b}}{N_{f}} \alpha_{1}, \quad \eta_{\phi}=\frac{\tilde{g}_{*}^{2}}{\tilde{g}_{\infty}^{2}} .
$$

The above $1 / N_{f}$ corrections are small, and about an order of magnitude smaller than for the purely relativistic case of Dirac fermions in $2+1$ dimensions [21]. In the latter case, the $1 / N_{f}^{2}$ corrections, computed at 2-loop order [33,34], are comparable or even larger than the $1 / N_{f}$ ones when $N_{f}=1$. For semi-Dirac fermions, on the other hand, at one-loop order, the $1 / N_{f}^{2}$ corrections are proportional to derivatives $F_{i}^{\prime}\left(\tilde{g}_{\infty}^{2}\right) \simeq$ $10^{-4}$ and hence about an order of magnitude smaller than the $1 / N_{f}$ ones. This suggests that the $1 / N_{f}$ expansion at the physical dimension is better controlled for the case of semi-Dirac fermions. However, the evaluation of two-loop diagrams would be required to investigate this further. 


\section{F. Critical exponents}

At the nontrivial critical fixed point the bosonic anomalous dimension $\eta_{\phi}=1+O\left(1 / N_{f}\right)$ is larger than the tree level scaling of the Yukawa coupling. Therefore, near the critical point $\tilde{g}$ flows toward $\tilde{g}_{*}$, making the fixed point stable along the $\tilde{g}$ axis. Near this multicritical point there are two relevant perturbations, $\Delta$ and $m_{\phi}^{2}$, which are the tuning parameters for the topological phase transition and the broken-symmetry state, respectively.

Semi-Dirac quasiparticle excitations emerge in the semimetallic phase when $\Delta$ is fine tuned to zero. Let us first consider the case that $\Delta$ remains equal to zero across the symmetry-breaking phase transition. In this case the RG equation for $m_{\phi}^{2}$ is equal to

$$
\frac{d m_{\phi}^{2}}{d \ell}=\left(2-\eta_{\phi}\right) m_{\phi}^{2} .
$$

The correlation length is defined by the RG scale $\ell^{*}$ at which $m_{\phi}^{2}\left(\ell^{*}\right) \simeq 1, \xi=e^{\ell^{*}}$. Integrating the above differential Eq. (51),

$$
m_{\phi}^{2}(\ell)=m_{\phi}^{2}(0) e^{\left(2-\eta_{\phi}\right) \ell}
$$

Using that $m_{\phi}^{2}(0) \sim\left(g_{c}-g\right)$, we obtain $\xi \sim\left(g_{c}-g\right)^{-v}$ with correlation length exponent $v=1 /\left(2-\eta_{\phi}\right)$. From Eq. (50), we obtain to order $1 / N_{f}$

$$
v=1-\frac{N_{b}}{2 N_{f}}\left(5 \alpha_{1}-\alpha_{2}\right)-2 \frac{2-N_{b}}{N_{f}} \alpha_{3} .
$$

The electronic dispersion of semi-Dirac fermions with linear and quadratic directions is strongly anisotropic. One therefore expects that the order parameter correlation inherits this anisotropy. For spatially isotropic systems, the correlations length along the imaginary-time direction diverges as a power of the spatial correlation length, $\xi_{\tau}=\xi^{z}$, where $z$ is the dynamical exponent. With our choice $z_{y}=1$, the dynamical exponent $z$ sets the scaling dimension of length along the $x$ direction. We therefore have $\xi_{\tau} \simeq \xi_{x} \simeq \xi_{y}^{z}$. The spatial anisotropy of the order parameter correlations is therefore reflected in correlation length exponents

$$
v_{x}=z v \quad \text { and } \quad v_{y}=v,
$$

along the $x$ and $y$ directions, respectively. In the limit $N_{f} \rightarrow \infty$ this gives $v_{x}=2$ and $v_{y}=1$.

Assuming that the system satisfies hyperscaling, we can use the standard scaling relations to obtain the remaining critical exponent. The Josephson hyperscaling relation yields the specific heat exponent,

$$
\begin{aligned}
\alpha & =2-v(2 z+1) \\
& \approx-3+\frac{N_{b}}{2 N_{f}}\left(21 \alpha_{1}-\alpha_{2}\right)+10 \frac{2-N_{b}}{N_{f}} \alpha_{3} .
\end{aligned}
$$

Note that the effective dimension that enters in the hyperscaling relation is equal to $D=2 z+1$ corresponding to one spacelike and two timelike directions. Fisher's scaling law gives the susceptibility exponent

$$
\gamma=\left(2-\eta_{\phi}\right) v=1+O\left(1 / N_{f}^{2}\right) .
$$

TABLE I. Critical exponents for symmetry-breaking phase transitions of semi-Dirac fermions in $2+1$ dimensions, calculated at one-loop order and including $1 / N_{f}$ corrections in the number of fermion flavors. $N_{b}$ is the number of order parameter components: $N_{b}=1$ for the CDW, $N_{b}=2$ for the superconducting, and $N_{b}=3$ for the SDW instabilities.

\begin{tabular}{cc}
\hline \hline Exponent & Value \\
\hline$z_{y}$ & 1 \\
$z$ & $2+0.0123 \frac{N_{b}}{N_{f}}$ \\
$\eta_{\psi}$ & $0.0125 \frac{N_{b}}{N_{f}}$ \\
$\eta_{\phi}$ & $1-0.0310 \frac{N_{b}}{N_{f}}-0.1069 \frac{2-N_{b}}{N_{f}}$ \\
$v$ & $1-0.0310 \frac{N_{b}}{N_{f}}-0.1069 \frac{2-N_{b}}{N_{f}}$ \\
$\alpha$ & $-3+0.1307 \frac{N_{b}}{N_{f}}+0.5345 \frac{2-N_{b}}{N_{f}}$ \\
$\gamma$ & 1 \\
$\beta$ & $2-0.0653 \frac{N_{b}}{N_{f}}-0.2672 \frac{2-N_{b}}{N_{f}}$ \\
$\delta$ & $\frac{3}{2}+0.0163 \frac{N_{b}}{N_{f}}+0.0668 \frac{2-N_{b}}{N_{f}}$ \\
\hline \hline
\end{tabular}

We can use Rushbrooke's scaling law $\alpha+2 \beta+\gamma=2$ to obtain the order parameter critical exponent

$$
\begin{aligned}
\beta & =1-\frac{1}{2}(\alpha+\gamma) \\
& \approx 2-\frac{N_{b}}{4 N_{f}}\left(21 \alpha_{1}-\alpha_{2}\right)-5 \frac{2-N_{b}}{N_{f}} \alpha_{3} .
\end{aligned}
$$

Finally, from the Widom identity $\gamma=\beta(\delta-1)$ we compute the field exponent

$$
\begin{aligned}
\delta & =1+\frac{\gamma}{\beta} \\
& \approx \frac{3}{2}+\frac{N_{b}}{16 N_{f}}\left(21 \alpha_{1}-\alpha_{2}\right)+\frac{5}{4} \frac{2-N_{b}}{N_{f}} \alpha_{3} .
\end{aligned}
$$

A complete list of critical exponents with numerical values for the coefficients $\alpha_{i}$ can be found in Table I.

\section{G. Multicriticality}

In Sec. III F we have summarized the universal critical behavior of semi-Dirac fermions associated with spontaneous symmetry breaking due to short-range interactions. The semiDirac quasiparticle excitations in the disordered, semimetallic phase are obtained by fine-tuning the system to the point of a topological phase transition between a Dirac semimetal with two separate relativistic Dirac points and a band insulator. In the free-fermion action (1) the semi-Dirac point corresponds to $\Delta=0$. Spontaneous symmetry breaking leads to the opening of a gap in the fermion spectrum, making it in practice challenging to ensure $\Delta=0$ across the transition in any real material.

Since the tuning parameters of the symmetry-breaking and topological phase transitions, $m_{\phi}^{2}$ and $\Delta$, are both relevant perturbations at the fixed point $\left(\tilde{g}=\tilde{g}_{*}, \Delta=m_{\phi}^{2}=0\right)$, one 
should view the fixed point as multicritical. The coupled RG equations for $m_{\phi}^{2}$ and $\Delta$ are

$$
\begin{aligned}
\frac{d m_{\phi}^{2}}{d \ell} & =\left(2-\eta_{\phi}\right) m_{\phi}^{2}+z \Pi_{m_{\phi}^{2}}, \\
\frac{d \Delta}{d \ell} & =\left(2-\eta_{\psi}\right) \Delta+z \Sigma_{\Delta},
\end{aligned}
$$

where $\eta_{\phi}$ and $\eta_{\psi}$ are the anomalous dimensions (50) of the fermions and bosons, respectively. The renormalizations $\Pi_{m_{\phi}^{2}}$ and $\Sigma_{\Delta}$ are coming from the bosonic self-energy (Fig. 2) and fermionic self-energy [Fig. 3(a)] and are equal to

$$
\begin{gathered}
\Pi_{m_{\phi}^{2}}=\frac{2}{3 \pi^{2}} \tilde{g}^{2} \Delta, \\
\Sigma_{\Delta}=\frac{N_{b}}{2 N_{f}}\left[F_{4}\left(\tilde{g}^{2}\right) \Delta+F_{5}\left(\tilde{g}^{2}\right) m_{\phi}^{2}\right] .
\end{gathered}
$$

In the last equation, we have defined the functions

$$
\begin{gathered}
F_{4}(x)=\frac{1}{\pi^{2}} \int_{0}^{\frac{\pi}{2}} d \theta \frac{\cos 2 \theta}{x^{-1} \cos \theta+\sqrt{\sin \theta}} \frac{\sin \theta}{\sqrt{\cos \theta}}, \\
F_{5}(x)=\frac{1}{\pi^{2}} \int_{0}^{\frac{\pi}{2}} d \theta \frac{\sqrt{\cos \theta} \sin \theta}{\left(x^{-1} \cos \theta+\sqrt{\sin \theta}\right)^{2}},
\end{gathered}
$$

which are shown in Fig. 4. The coupled RG equations are of the form

$$
\frac{d}{d \ell}\left(\begin{array}{c}
m_{\phi}^{2} \\
\Delta
\end{array}\right)=\mathbf{M}\left(\begin{array}{c}
m_{\phi}^{2} \\
\Delta
\end{array}\right)
$$

where $\mathbf{M}$ is an off-diagonal matrix that we evaluate at the multicritical fixed point. The two positive eigenvalues $\theta_{1}$ and $\theta_{2}$ of this matrix are inversely proportional to correlation length exponents, $v_{i}=1 / \theta_{i}$. Up to order $1 / N_{f}$ we obtain

$$
\begin{gathered}
v_{1}=1-\frac{N_{b}}{2 N_{f}}\left(5 \alpha_{1}-\alpha_{2}-\frac{28}{11} \alpha_{5}\right)-2 \frac{2-N_{b}}{N_{f}} \alpha_{3}, \\
v_{2}=\frac{1}{2}+\frac{N_{b}}{4 N_{f}}\left(\alpha_{1}-\alpha_{4}-\frac{14}{11} \alpha_{5}\right) .
\end{gathered}
$$

\section{MEAN-FIELD ANALYSIS}

The critical exponents are expected to recover the meanfield values in the limit $N_{f} \rightarrow \infty$. In this limit, the anomalous dimension of the fermion fields vanishes, $\eta_{\psi}=0$, indicating that Fermi-liquid behavior is recovered. However, the anomalous dimension of the order parameter field (50) remains finite, $\eta_{\phi}=1$. This results in a correlation length exponent $v=1 /\left(2-\eta_{\phi}\right)=1$ and, using standard scaling and hyperscaling relations, in an unusually large order parameter exponent $\beta=2$. These exponents are very different from the usual mean-field ones $\left(\eta_{\phi}=0, v=\beta=\frac{1}{2}\right)$. This unusual behavior is a result of the appearance of nonanalytic terms in the mean-field free energy, which lead to unconventional quantum criticality and arise due to the unusual scaling of the density of states $\rho(\epsilon) \sim \sqrt{\epsilon}$ around the Fermi points.

The mean-field free energy for a gapped phase of semiDirac fermions is equal to

$$
\mathcal{F}_{\mathrm{MF}}\left(\phi_{0}\right)=\frac{1}{g} \phi_{0}^{2}-\int_{k_{x}^{2}+k_{y}^{4} \leqslant \Lambda^{2}} \frac{d^{2} \boldsymbol{k}}{(2 \pi)^{2}} \sqrt{k_{x}^{2}+k_{y}^{4}+\phi_{0}^{2}},
$$

where we have rescaled $k_{x}$ and $k_{y}$ to absorb the prefactors $v$ and $1 /(2 m)$. Here $\Lambda$ is the UV energy cutoff. Carrying out the integral one obtains [28]

$$
\mathcal{F}_{\mathrm{MF}}\left(\phi_{0}\right)=a(\delta g) \phi_{0}^{2}+b\left|\phi_{0}\right|^{\frac{5}{2}}+O\left(\phi_{0}^{4}\right)
$$

with $\delta g=\left(g_{c}-g\right) / g_{c}$ and $a, b>0$. As in the case of relativistic Dirac fermions, the mean-field free energy contains a nonanalytic term, $\left|\phi_{0}\right|^{\frac{5}{2}}$, which arises from the evaluation of the integral in the $\boldsymbol{k} \rightarrow 0$ limit. Minimizing $\mathcal{F}_{\mathrm{MF}}\left(\phi_{0}\right)$ with respect to $\phi_{0}$ one obtains $\left|\phi_{0}\right| \sim|\delta g|^{\beta_{\mathrm{MF}}}$ with $\beta_{\mathrm{MF}}=2$, in agreement with the RG and scaling analysis in the $N_{f} \rightarrow \infty$ limit, Eq. (57). In contrast, for Dirac fermions, where the density of states vanishes linearly $[\rho(\epsilon) \sim \epsilon]$, the nonanalytic term has the form $\left|\phi_{0}\right|^{3}$, resulting instead in the mean-field exponent $\beta_{\mathrm{MF}}=1$ [35].

The spatial anisotropy of the system, which appears in the anisotropic dispersion of the quasiparticles, also reflects in the form of the gradient terms in the Ginzburg-Landau functional. These terms can be computed by allowing for small, long-wavelength modulations of the order parameter. For a finite homogeneous component $\phi_{0}$ one can expand in the momentum $\boldsymbol{q}$ of the modulation. This gives rise to terms $q_{x}^{2} \sqrt{\left|\phi_{0}\right|}$ and $q_{y}^{2}\left|\phi_{0}\right|^{\frac{3}{2}}$ [28], from which we can estimate the correlation lengths $\xi_{x}$ and $\xi_{y}$ along the $x$ and $y$ directions, respectively. Since

$$
\xi_{x}^{-2}\left|\phi_{0}\right|^{\frac{1}{2}} \simeq \xi_{y}^{-2}\left|\phi_{0}\right|^{\frac{3}{2}} \simeq|\delta g| \phi_{0}^{2}
$$

by dimensional analysis, that leads to the quantum critical scaling

$$
\xi_{x}^{2} \sim\left|\phi_{0}\right|^{-\frac{3}{2}}|\delta g|^{-1} \sim|\delta g|^{-\left(1+\frac{3}{2} \beta_{\mathrm{MF}}\right)}
$$

and

$$
\xi_{y}^{2} \sim\left|\phi_{0}\right|^{-\frac{1}{2}}|\delta g|^{-1} \sim|\delta g|^{-\left(1+\frac{1}{2} \beta_{\mathrm{MF}}\right)} .
$$

Using $\beta_{\mathrm{MF}}=2$, this simple scaling analysis of the mean-field free energy recovers the correlation length exponents $v_{x}=2$ and $v_{y}=1$ derived in Eq. (54), in agreement with the RG result in the limit $N_{f} \rightarrow \infty$.

The anisotropic scaling of the correlation length along the $x$ and $y$ directions could have very interesting implications for ordered phases in the vicinity of the quantum critical point. In general, the order parameter becomes relatively softer to spatial modulations along the direction where the quasiparticles have parabolic dispersion, and more rigid in the other direction, permitting the emergence of modulated order and stripe phases [28]. In the superconducting case, the system may effectively respond to a external magnetic field as a type II superconductor in one direction and as a type I in the other [28]. This unconventional state could stabilize stripes of magnetic flux rather than conventional vortex lattices.

\section{DISCUSSION}

We have analyzed the critical behavior of quantum phase transitions in semi-Dirac fermion systems that are driven by strong short-range interactions. Here we have focused on SDW, CDW, and superconducting instabilities that fully gap the quasiparticle spectrum in the broken-symmetry state. 
Those instabilities are generically expected to be energetically favorable compared to gapless states.

This criticality was previously studied using an $\epsilon$ expansion in the number of quadratically dispersing directions within the Yukawa formalism [27] and by generalizing the quadratic dispersion to $k_{y}^{2 n}$, facilitating a $1 / n$ expansion in generic four-fermion interactions around the one-dimensional limit [26]. In our complementary approach, we have avoided the tuning of dimensionality but instead introduced a large number $N_{f}$ of fermion flavors for analytic control in two spatial dimensions. Using a one-loop renormalization group analysis of the effective Yukawa actions, we have computed critical exponents up to order $1 / N_{f}$.

The $1 / N_{f}$ corrections to critical exponents depend on the peculiar form of the IR order parameter propagator in $2+1$ dimensions, $G_{\phi}^{\mathrm{IR}} \sim\left(q_{0}^{2}+v^{2} q_{x}^{2}\right)^{-\frac{1}{4}}$. This IR contribution, which is not renormalized by integrating out electronic UV modes, needs to be incorporated to regularize unphysical divergencies [25]. Our RG equations are derived from integrating UV modes from an infinitesimal $2+1$ dimensional shell in momentum-frequency space, followed by a rescaling of frequency and momenta to the original cutoff. We have treated frequency $k_{0}$ and the momentum $k_{x}$ along the relativistic direction on an equal footing and introduced a single "dynamical" exponent $z$ that describes the scaling of $k_{0}, k_{x}$ relative to the quadratically dispersing direction $k_{y}$. One might view this as having one spacelike and two timelike directions.

We have found that the $1 / N_{f}$ corrections to critical exponent are smaller than for the case of Dirac fermions and seem to fall off more rapidly when increasing the order of $1 / N_{f}$. This suggests that the $1 / N_{f}$ expansion is well controlled even when the number of flavors $N_{f}$ is of the order of 1 . However, calculations beyond one-loop order are required to confirm this conjecture.

In the mean-field limit $N_{f} \rightarrow \infty$, the anomalous dimension $\eta_{\psi}$ of the fermion fields vanishes, signaling a recovery of conventional Fermi-liquid behavior. On the other hand, the anomalous dimension of the order parameter fields remains finite, $\eta_{\phi}=1$. This has important consequences. It gives rise to a correlation length exponent of $v=1$ instead of the conventional mean-field $v=1 / 2$. Since we have defined the $y$ direction as reference length $\left(z_{y}=1, z_{x}=z=2\right)$, this corresponds to $v_{y}=z_{y} v=1$ and $v_{x}=z_{x} v=2$ along the two spatial directions.

The atypical correlation length exponent and the unusually large order parameter exponent $\beta=2+O\left(1 / N_{f}\right)$ suggest that the mean-field order parameter theory is highly unusual [28]. As we discussed, the vanishing density of states at the Fermi level gives rise to a nonanalytic $\left|\phi_{0}\right|^{5 / 2}$ term in the Landau free energy. This results in $\beta_{\mathrm{MF}}=2$, in agreement with the
RG result for $N_{f} \rightarrow \infty$. The highly anisotropic order parameter correlations correspond to different nonanalytic gradient terms in the mean-field theory.

We now briefly compare to previous results $[26,27]$ in the literature. The freedom in how to define the scaling dimensions in semi-Dirac systems explains the apparent contradiction with Ref. [26] that reports $v=2$ at leading order, compared to $v=1$ here and in Ref. [27]. A close inspection shows that in this work the relativistic direction was used to define the reference length scale. In our notation this corresponds to the choice $z=z_{x}=1$ and $z_{y}=1 / 2$, leading to the same correlation length exponents $v_{x}=2$ and $v_{y}=1$. Hence, to leading order there is complete agreement on the correlation length exponent.

However, there is a strong disparity in the order parameter anomalous dimension found here $\eta_{\phi}=1+O\left(1 / N_{f}\right)$, and in Ref. [27], $\eta_{\phi} \sim \frac{1}{N_{f}} \epsilon \ln \epsilon$. Not only does the latter vanish for $\epsilon=1$, but it is also of order $1 / N_{f}$. As discussed in Sec. IV, our result $\eta_{\phi} \approx 1$ leads to the order parameter exponent $\beta=$ 2 in the $N_{f} \rightarrow \infty$ limit, in complete agreement with the nonanalytic mean-field free energy. This acts as a consistency check of our RG analysis. Likewise, the fermion anomalous dimension $\eta_{\psi} \sim \frac{1}{N_{f}} \epsilon \ln \epsilon$ calculated in Ref. [27] vanishes at $\epsilon=1$, whereas ours is finite $\eta_{\psi}=O\left(1 / N_{f}\right)$. These discrepancies may arise from the lack of validity in the extrapolation of their $\epsilon$ expansion to the physical dimension at $\epsilon=1$. We also note that at the leading one-loop order it is not possible to extract the anomalous dimensions from the RG analysis of the model containing four-fermion interactions studied in Ref. [26]. In the future it will be interesting to compare beyond leading order results for the critical exponents in this difficult problem.

Semi-Dirac fermions correspond to an intermediate case between Dirac fermions and ordinary metals in two spatial dimensions. The quadratic dispersion along one of the momentum directions leads to an increased density of states at low energies as compared to Dirac fermions, making instabilities comparatively easier due to the enlarged phase space for quantum fluctuations. An interesting question for future studies is whether the enhanced electronic fluctuations near the quantum critical point combined with the anisotropy of the correlations could stabilize novel phases such as modulated order.

\section{ACKNOWLEDGMENTS}

We thank A. Green and G. Jose for useful discussions. B.U. acknowledges NSF CAREER Grant No. DMR-1352604 for partial support. F.K. acknowledges financial support from EPSRC under Grant No. EP/P013449/1.
[1] A. H. Castro Neto, F. Guinea, N. M. R. Peres, K. S. Novoselov, and A. K. Geim, Rev. Mod. Phys. 81, 109 (2009).

[2] X.-L. Qi and S.-C. Zhang, Rev. Mod. Phys. 83, 1057 (2011).

[3] M. Z. Hasan and C. L. Kane, Rev. Mod. Phys. 82, 3045 (2010).

[4] N. P. Armitage, E. J. Mele, and A. Vishwanath, Rev. Mod. Phys. 90, 015001 (2018).
[5] P. Dietl, F. Piéchon, and G. Montambaux, Phys. Rev. Lett. 100, 236405 (2008).

[6] G. Montambaux, F. Piéchon, J.-N. Fuchs, and M. O. Goerbig, Phys. Rev. B 80, 153412 (2009).

[7] S. Banerjee, R. R. P. Singh, V. Pardo, and W. E. Pickett, Phys. Rev. Lett. 103, 016402 (2009). 
[8] A. S. Rodin, A. Carvalho, and A. H. Castro Neto, Phys. Rev. Lett. 112, 176801 (2014).

[9] J. Kim, S. S. Baik, S. H. Ryu, Y. Sohn, S. Park, B.-G. Park, J. Denlinger, Y. Yi, H. J. Choi, and K. S. Kim, Science 349, 723 (2015).

[10] J. Kim, S. S. Baik, S. W. Jung, Y. Sohn, S. H. Ryu, H. J. Choi, B.-J. Yang, and K. S. Kim, Phys. Rev. Lett. 119, 226801 (2017).

[11] S. Katayama, A. Kobayashi, and Y. Suzumura, J. Phys. Soc. Jpn. 75, 054705 (2006).

[12] V. Pardo and W. E. Pickett, Phys. Rev. Lett. 102, 166803 (2009).

[13] H. Huang, Z. Liu, H. Zhang, W. Duan, and D. Vanderbilt, Phys. Rev. B 92, 161115(R) (2015).

[14] E. Christou, B. Uchoa, and F. Krüger, Phys. Rev. B 98, 161120(R) (2018).

[15] E. Christou, F. de Juan, and F. Krüger, arXiv:1906.03892.

[16] J. M. Link, B. N. Narozhny, E. I. Kiselev, and J. Schmalian, Phys. Rev. Lett. 120, 196801 (2018).

[17] V. N. Kotov, B. Uchoa, V. M. Pereira, F. Guinea, and A. H. Castro Neto, Rev. Mod. Phys. 84, 1067 (2012).

[18] I. F. Herbut, Phys. Rev. Lett. 97, 146401 (2006).

[19] I. F. Herbut, V. Juričić, and O. Vafek, Phys. Rev. B 80, 075432 (2009).
[20] F. F. Assaad and I. F. Herbut, Phys. Rev. X 3, 031010 (2013).

[21] L. Janssen and I. F. Herbut, Phys. Rev. B 89, 205403 (2014).

[22] D. J. Gross and A. Neveu, Phys. Rev. D 10, 3235 (1974).

[23] J. Zinn-Justin, Nucl. Phys. B 367, 105 (1991).

[24] G. Y. Cho and E.-G. Moon, Sci. Rep. 6, 19198 (2016).

[25] H. Isobe, B.-J. Yang, A. Chubukov, J. Schmalian, and N. Nagaosa, Phys. Rev. Lett. 116, 076803 (2016).

[26] B. Roy and M. S. Foster, Phys. Rev. X 8, 011049 (2018).

[27] S. Sur and B. Roy, arXiv:1812.05615.

[28] B. Uchoa and K. Seo, Phys. Rev. B 96, 220503(R) (2017).

[29] B.-J. Yang, E.-G. Moon, H. Isobe, and N. Nagaosa, Nat. Phys. 10, 774 (2014).

[30] X. Li, J.-R. Wang, and G.-Z. Liu, Phys. Rev. B 97, 184508 (2018).

[31] S. E. Han, G. Y. Cho, and E.-G. Moon, Phys. Rev. B 98, 085149 (2018).

[32] S. E. Han, C. Lee, E.-G. Moon, and H. Min, Phys. Rev. Lett. 122, 187601 (2019).

[33] A. N. Vasil'ev, S. É. Derkachev, N. A. Kivel', and A. S. Stepanenko, Theor. Math. Phys. 94, 127 (1993).

[34] J. Gracey, Int. J. Mod. Phys. A 9, 727 (1994).

[35] S. Sachdev, Quantum Phase Transitions, 2nd ed. (Cambridge University Press, Cambridge, 2011). 